\title{
Architecture neural network deep optimizing based on self organizing feature map algorithm
}

\author{
Muthna Jasim Fadhil, Majli Nema Hawas, Maitham Ali Naji \\ Department of Electrical Power Eng., Electrical Engineering Technical College, \\ Middle Technical University (MTU), Iraq
}

\begin{tabular}{l} 
Article Info \\
\hline Article history: \\
Received Nov 26, 2019 \\
Revised Mar 23, 2020 \\
Accepted Apr 4, 2020 \\
\hline Keywords: \\
Architecture for optimization \\
Hidden layers \\
Hidden neurons \\
Neural network \\
Self organizing feature map
\end{tabular}

\begin{abstract}
Forward neural network (FNN) execution relying on the algorithm of training and architecture selection. Different parameters using for nip out the architecture of FNN such as the connections number among strata, neurons hidden number in each strata hidden and hidden strata number. Feature architectural combinations exponential could be uncontrollable manually so specific architecture can be design automatically by using special algorithm which build system with ability generalization better. Determination of architecture FNN can be done by using the algorithm of optimization numerous. In this paper methodology new proposes achievement where FNN neurons respective with hidden layers estimation work where in this work collect algorithm training self organizing feature map (SOFM) with advantages to explain how the best architectural selected automatically by SOFM from criteria error testing based on architecture populated. Different size of dataset benchmark of 4 classifications tested for approach proposed.
\end{abstract}

This is an open access article under the CC BY-SA license.

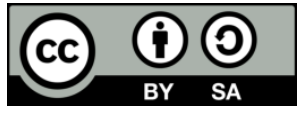

\section{Corresponding Author:}

Muthna Jasim Fadhil,

Electrical Engineering Technical College,

Middle Technical University (MTU),

Al-Doora, Al-Masafee street, Baghdad, Iraq.

Email: muthnafadhil@gmail.com

\section{INTRODUCTION}

Information process of brain human way mimic designed by mathematical model of artificial neural network (ANN) where divided into hidden, output and input layers. In any neural network layers of hidden represent counting engine, the popularity of ANN coming from problems complexity solving capability and ability learning good and design simple [1-3]. Neurons units processing number in model of ANN for layers hidden variables while these constant for layers input and output. Criterion straightforward involve to determine neurons number in layers hidden and for calculating theory supporting found for hidden layers. These architecture assign implicated with execution of ANN because under fitting comes from less neurons and less layers network while over fitting caused by massive network. Also, ANN that have various formation gives various output to itself set data so ANN design architecture is decisive and could be relate as problem optimization $[4,5]$.

Optimization ANN architectures populated known as solutions and the cost function represent their experimentation error. Thus, the defy is to obtain most favorable architecture with minimum error testing via several improvement techniques. Generally, ANN architecture choice depending on slap and test attitude, which is time unbearable and challenges many poses, such as connections, hidden neurons, hidden layers primary number of etc. Functioning ANN pre-knowledge required to solve dominant problem [6-9]. These primary 
parameters could not be integration without any knowledge because the attributes may be combinations in exponentially. Thus, it needs highly work hard intensive human when using test and hit method for parameters selecting without guarantee to get exact model. Additional, when domain complexity produce problem then resolve ANN parameters becomes relatively complex and dreary process. Algorithms optimization widely using to handle the following issues; includingand bat algorithm, annealing simulated and genetic algorithm [10].

Many algorithms using in structure ANN optimal nearest or optimal suggest which are ANN architecture as well as rule training optimizing proposed for the last decades. Xia and Kamel [11] technique optimization stochastic as algorithm pruning based SA and GA integration applied. Jiaying et al. [12] multilayer perceptionoptimize by using algorithm based SA and TS proposed where low convergence avoids iteration single solution batch evaluates Janssens [13] architecture ANN optimize using process evolutionary multi object employed where problems real word twice face recognition and classification car processing by TS with SA integrates. Al-Kazemi and Mohan [14] using algorithm genetic Tagushi hybrid to assist in FNN parameter design based ANN (PSO) Practical swarm optimization proposed design parameters and architecture FNN three layer evaluated in PSO discrete and PSO version improved.

In this paper the advantages combines methodology new proposed for bothandSOFM training algorithm for ruling best design of FNN where neurons respective their hidden layers defining problems handles automatically which represent manual task for cases earlier. Methodology application training in four various sets data classification: 1. Dataset ISOLET [15], 2. Digits handwritten dataset MNIST [14], 3. Dataset drift array sensor gas [5], 4. Dataset face recognition [7]. The paper planned as: verious techniques optimization using optimization FNN on works related describes section 2. Representation solution like components used methodology optimization presents, mechanism stopping, generation population and function fitness section 3. Study that used properties of data sets describes section 4. Results and setup experimental present section 5. Finally, future scope and discussion covers the paper section 6.

\section{METHODOLOGY OPTIMIZATION}

SOFM withalgorithm effective knowledge domains problem diverse in consideration abroad because of optimal solution globally finding in achievement considerable and adaptability. Neural network forming by connected multiple layer, each two neurons connection represent strength and activation of FNN. The output and input layer represent place stop and start respectively then between these two layers find hidden layer which minimizing error between input and output by adjustment weights as shown in Figure 1.

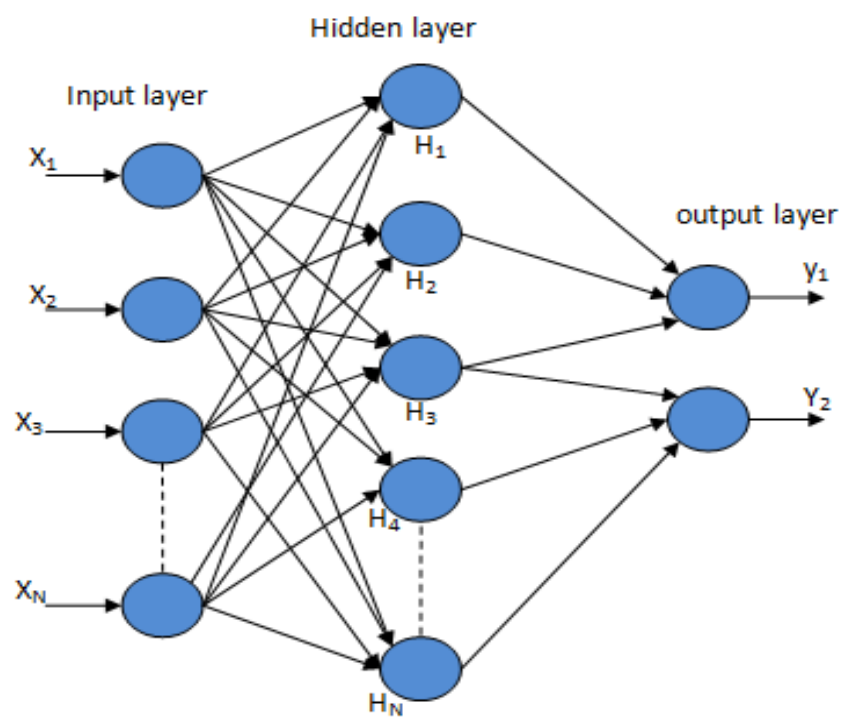

Figure 1. Architecture neural network

The neighbourhood search using variation memories short and long via methods of heuristic and locallysearch while the method of SOFM withstrategy convergence faster tends and cost computational minimizes which iteration single solution batch determination [16-18]. Next iteration of solution currently accepted and the final iteration given lowest cost which represent the best solution where the reptilian avoid 
and the final solution visited records which tabu list maintains strategy. The convolution layer comes before layer of sub sampling and planes number same ofconvolution layers number. Map feature size reduce to the desired layer by information relative preserves layer sub sampling between exact relation performance and features map SOFM, Figure 2 explain processing of working layer sub sampling.

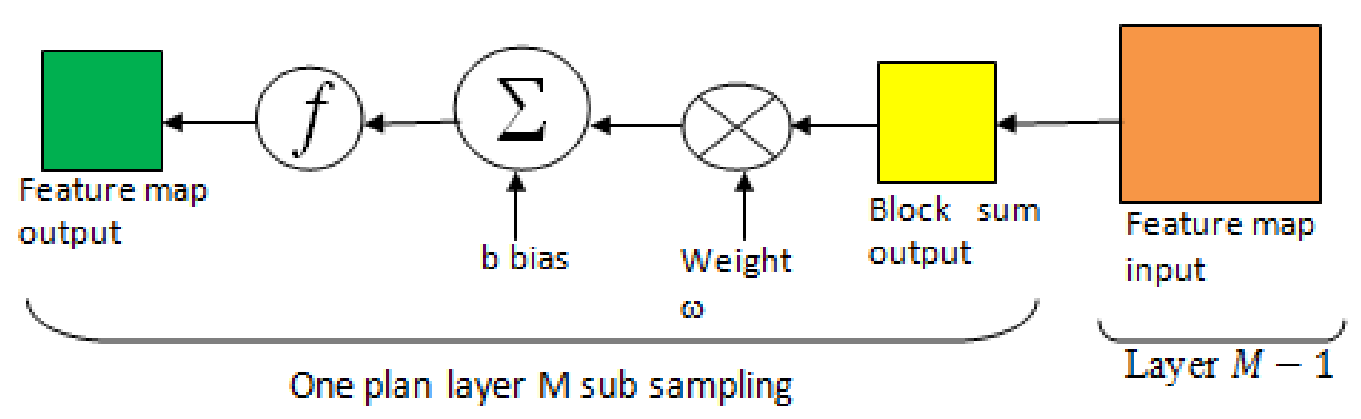

Figure 2. Processing of working layer sub sampling

In this paper, SOFM integrated withto reach optimization aim to find $\mathrm{r}$ solution by searching in $\mathrm{R}$ solution set where $f(r) \leq f\left(r^{\prime}\right)$, for all $r^{\prime} \in R$. Neurons hidden selected randomly and hidden layer having FNN with starts methodology, methodology proposed flow chart shows in Figure 3 where methodology proposed processing to maximum by means hidden layers max. Iter times iterated gets a solution, Pmax size population generates each iteration and fitness function based on best choice of SOFM selection. If the Iter (instruction) is best represented by $r^{\prime}$ and consequntly $r$ value more than $r^{\prime}$ so $r$ is best update and iteration next run. Or else list_Tabu update and ensure criteria not stopping by $r^{\prime}$ explore further [18-20]. Figure 4 represent methodology proposed pseudo code algorithm1SOFM withconsider the following strategy proposed implementation:

a. Representation solution.

b. Function fitness.

c. Generation population.

d. Mechanism stopping.

\subsection{Representation solution}

Evaluation considered various hidden layer with FNN, contains each network nodes output Q, nodes hidden $\mathrm{Kj}$ at $\mathrm{jth}$ hidden layers and nodes input $\mathrm{D}$. Usually specific problem is nodes output and input number where nodes respective and hidden layers number of optimal find, FNN architecture form as [21-23]:

$$
\mathrm{O} \equiv\left(\mathrm{D} \times \mathrm{K}_{1}+\mathrm{P} \times \mathrm{K}_{1}\right)+\left(\mathrm{K}_{1} \times \mathrm{K}_{2}+\mathrm{P} \times \mathrm{K}_{2}\right)+\cdots \ldots+\left(\mathrm{K}_{\max } \times \mathrm{Q}+\mathrm{P} \times \mathrm{Q}\right)
$$

Each solution contains from three variables: KL, consist of hidden layers number, KM is a vector consist of neurons hidden number, ET is solution given error of testing and P represent bias.

$$
\begin{aligned}
& \mathrm{R} \equiv\left(\mathrm{K}_{\mathrm{L}}, \mathrm{K}_{\mathrm{M}}, \mathrm{E}_{\mathrm{T}}\right) \\
& \mathrm{K}_{\mathrm{M}} \equiv\left(\mathrm{K}_{1}, \mathrm{~K}_{2}, \mathrm{~K}_{3}, \ldots, \mathrm{K}_{\max }\right), \mathrm{K}_{\mathrm{F}} \in \mathrm{M}, \forall \mathrm{K}_{(\mathrm{F}-1)}>\mathrm{K}_{\mathrm{F}}>\mathrm{K}_{(\mathrm{F}+1)}
\end{aligned}
$$

And $\mathrm{F}=1,2, \ldots, \max$.

$$
\mathrm{K}_{\mathrm{L}}=(1,2,3, \ldots, \max ) \text { and } \mathrm{E}_{\mathrm{T}} \in \mathrm{S}
$$

Where $\mathrm{S}$ is real numbers set and $\mathrm{M}$ is natural numbers set. Hidden layer single with neurons output and input number fixed consist of FNN connected fully which represent initial solution. The range between [(D+Q)/2, $(\mathrm{D}+\mathrm{Q}) \times 2 / 3$ ] selection random using to determine neurons hidden and distributed uniformly interval range $[+1,-1]$ represent initial weights [24]. 


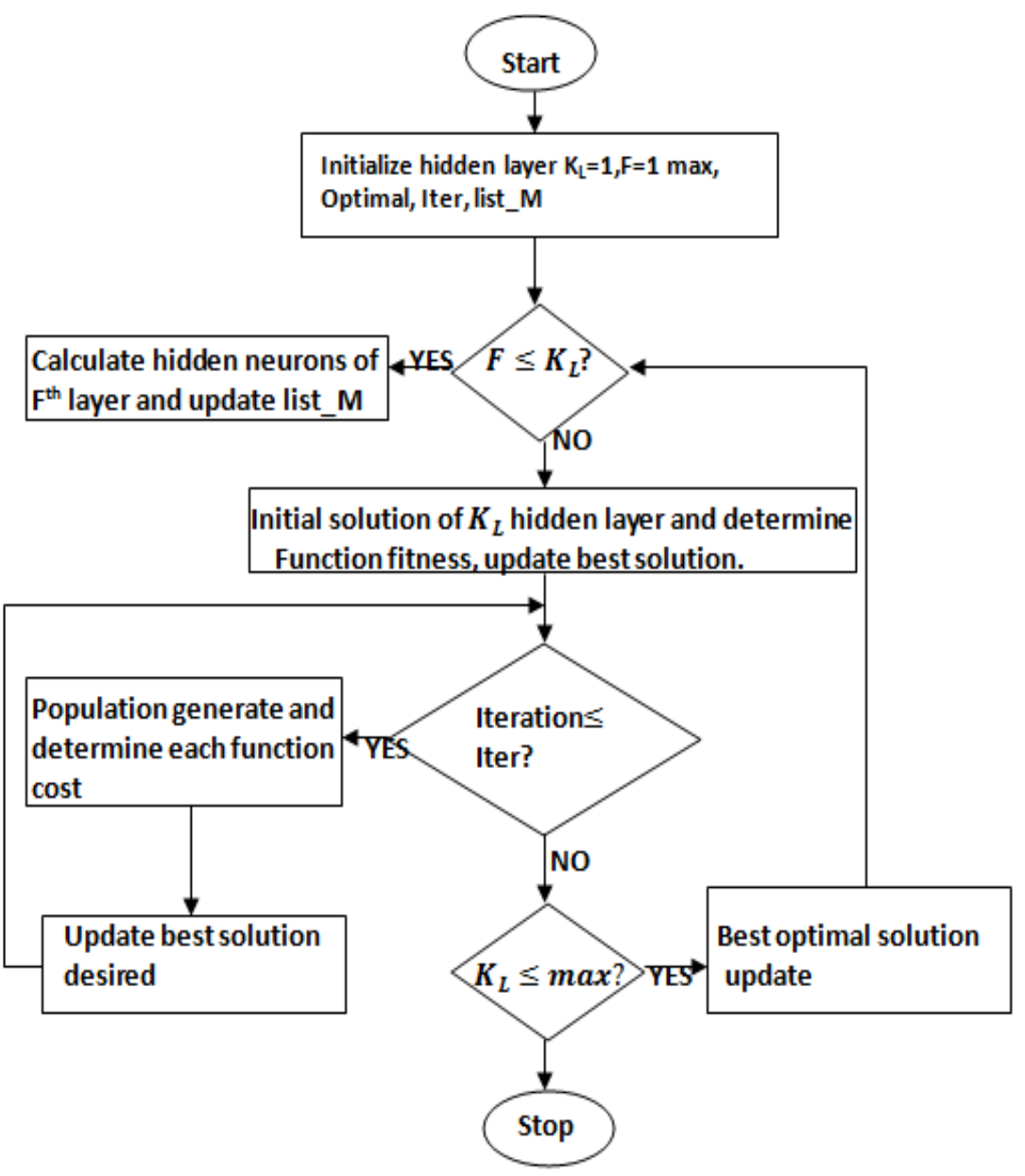

Figure 3. Algorithm proposed flow chart

\subsection{Function fitness}

The results approximation during capability terms model given accuracy percentage of function fitness where the function objective minimizes solution selected to iterations successive solution performance of its comparing requires. GM represent the classes after divided dataset and $\mathrm{E}$ set testing from $\mathrm{h}$ sample of actual class can be represented by [25-27]:

$$
\gamma(\mathrm{h}) \in\{1,2,3, \ldots, \mathrm{GM}\} \forall \mathrm{h} \in \mathrm{E}
$$

Correspondence one to one has dataset given GM classes and neurons output number using method of takes winner technique proposed, the $\mathrm{h}$ sample of $\mathrm{B}$ node output given the value $\mathrm{QB}(\mathrm{h})$ where $\mathrm{h}$ sample class is:

$$
\partial(\mathrm{h}) \equiv \operatorname{maxB} \arg \in[1,2, \ldots, \mathrm{GM}] \mathrm{QB}(\mathrm{h}) \forall \mathrm{h} \in \mathrm{E}
$$

And the $\mathrm{h}$ sample classes is:

$$
\epsilon(h) \equiv\left[\begin{array}{l}
1 \text { if } \gamma(h) \neq \partial(h) \\
0 \text { if } \gamma(h)=\partial(h)
\end{array}\right]
$$

So the percentage terms in phase testing during samples misclassified by means E set testing for network error classification represented by:

$$
\mathrm{T}(\mathrm{E}) \equiv \frac{100}{\mathrm{E}} \sum_{\mathrm{h} \in \mathrm{E}} \epsilon(\mathrm{h})
$$

Where E represent set testing cardinality. 


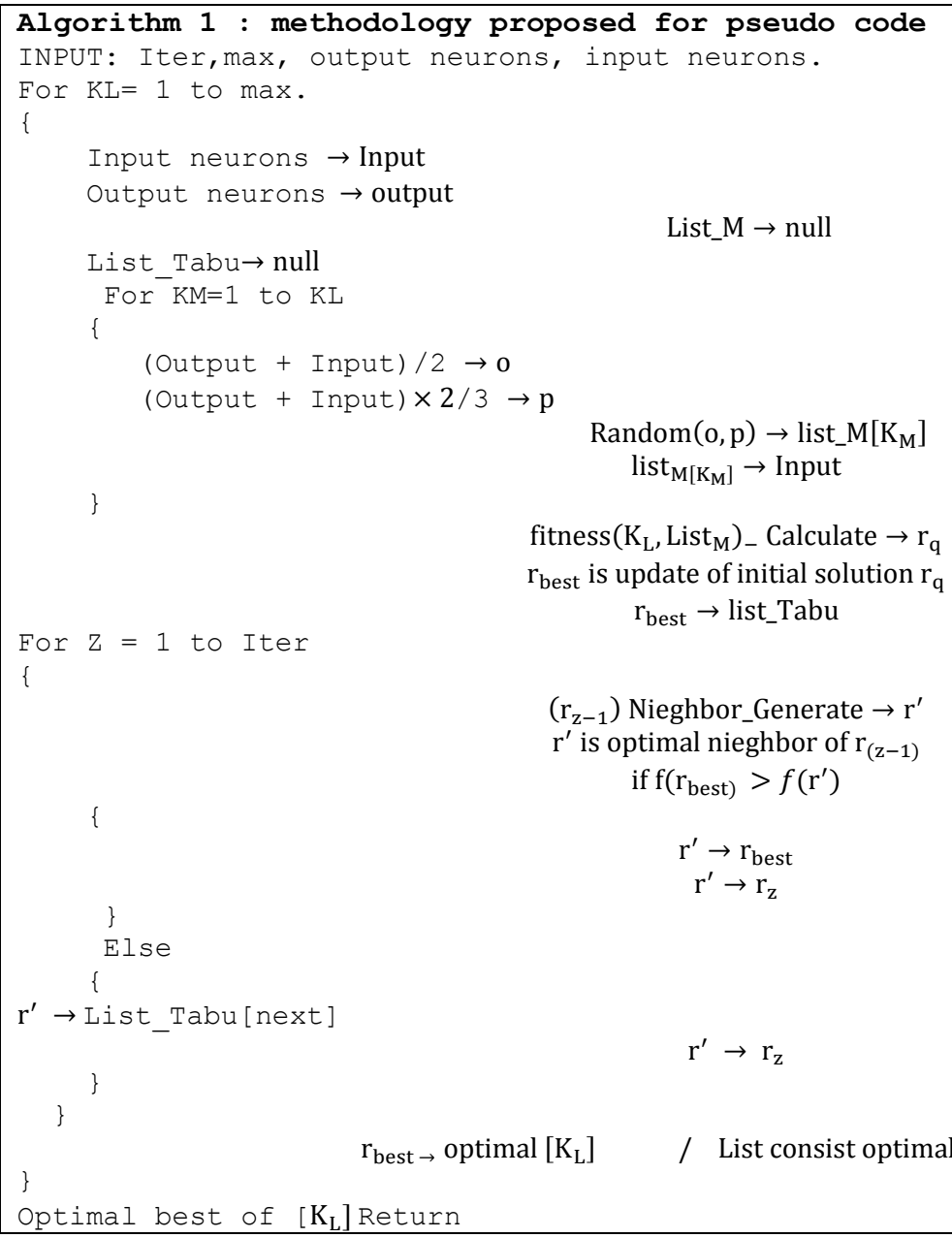

Figure 4. Methodology proposed pseudo code algorithm1

\subsection{Generation population}

Populates methodology proposed $\mathrm{r}=(\mathrm{KL}, \mathrm{KM}, \mathrm{ET})$ of initial solution evaluation to meet criteria stopping by using SOFM algorithm for solution generation complete where generated population new solution by population size divided in to equal 2 parts for layer particular neurons one for decreasing and other for increasing as shown in Figure 5. Case1: number random generate $\mathrm{x}$ of $[0,1]$ distributed uniformly for every layer $[17,28]$.

$$
\mathrm{KM}=\left\{\begin{array}{ll}
\omega+, & \mathrm{x} \geq \mathrm{p} \\
\text { no change } & \mathrm{x}<p
\end{array}\right\}
$$

Where w percentage neurons number increase by signifies $\omega+$ and continue until the rate don't go beyond high level boundary. Case2: $x$ number random generates:

$$
K M=\left\{\begin{array}{ll}
\omega- & x \geq B \\
\text { No change } & x<B
\end{array}\right\}
$$

Where $\omega$ percentage neurons number increase by signifies $\omega-$ until lower boundary reached. Solution optimal global search in directions backward and forward move possible it makes solutions new generating process.

\subsection{Mechanism stopping}

When iterations Iter reached to maximum hidden layers optimization that is guide to stop run SOFM algorithm. Step successive jumps and neurons updating avoids in some cases, in 1st step neurons number increases under processing SOFM algorithm and it arrive higher level already. The 2nd step neurons number decrease under processing SOFM algorithm and it arrive lower limit already. 


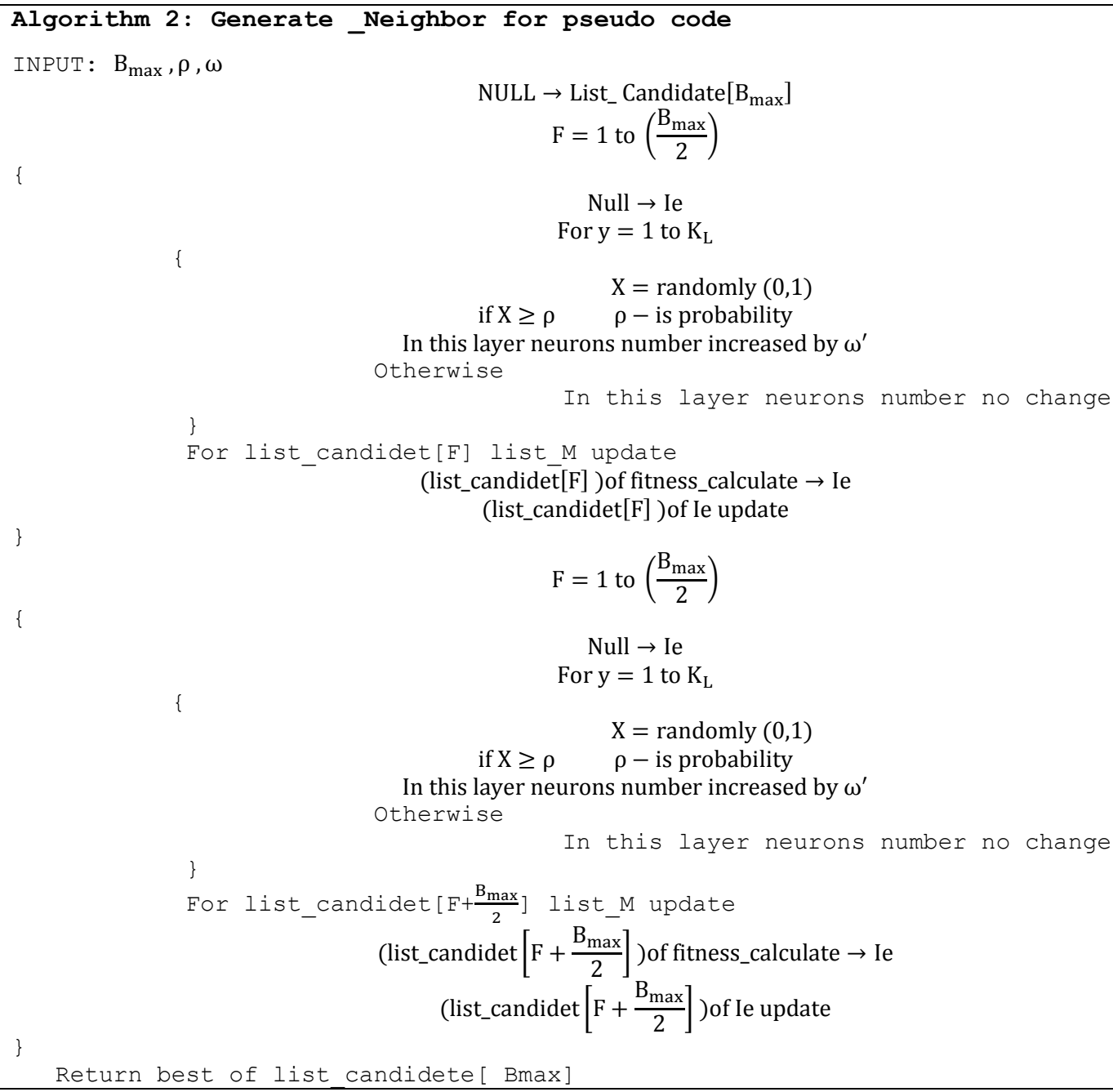

Figure 5. Algorithm using for solutions generation populates of methodology proposed

\section{DATASETS}

Algorithm proposed validate as shown in Table 1 using datasets classification in 4 divers features with huge number having datasets requires because the efficiency of algorithm would be low if processing in few features and in hidden layer single converge.

\subsection{Datasets face recognition}

The datasets using in this containing images of female and male with high resolution ages between 17-45 years including emotions different taken from Chicago University developed by Database Face Chicago (DFC) and each image formatted in JPEG, female are 878 and 974 males with total images 1852. The images changed to format vector from format JPEG, 1x785 dimension vector each label defines column last and image features describes vector every 784 to 1 column.

\subsection{Dataset drift array sensor gas}

Sixteen sensors chemical applied to 13920 examples of holds datasets concentrations levels different six gases task classification in compensation drift recreations employed where from involvement human caused errors common minimizing for environment computerized fully in platform delivery gas given as collected samples. Six outputs and 130 inputs has dataset, the performance good accomplish is a purpose for all time.

\subsection{Digits handwritten of dataset TSINM}

The TSINM (Technology Standards Institute National Modified) is large database consist of digits handwritten usually used in systems processing image that have many training, datasets TSIN samples using 
for generated database. The box of pixel $28 \times 28$ in normalized and formatted in bi-level for each image. The dataset TSINM consist of 70000 samples, outputs 10 and inputs 784, the range (0-9) using for classes distinct 10 in each image classified.

\subsection{Dataset LETISO}

LETISO (recognition speech letter isolated) dataset, twice alphabet each spoke by 150 speakers which distributed by author to get five groups, it classified as testing in one group, purpose training selected from four group dataset and thirty speakers in each group. The samples 7797 total dataset classes distinct 26 classified needs features 617 samples recorded.

\section{RESULTS AND EXPERIMENTAL SETUP}

Implementation SOFM with algorithm proposed, each architecture network selected randomly validated, set validation dataset of $20 \%$. The implementation based on function activation dropout which about 0.2 ratio dropout input and by method min-max normalized datasets. Table 1 datasets four classification tested is methodology proposed effectiveness. paper main involvement to get architecture optimal when datasets of FNN deep have features a huge number and needed in excess of 1 hidden layer.

Table 1. Statistics of dataset

\begin{tabular}{ccccc}
\hline References & classes & Features & Examples & Dataset \\
\hline$[15]$ & 26 & 617 & 7797 & LETISO \\
{$[14]$} & 10 & 784 & 70000 & TSINM \\
{$[5]$} & 6 & 130 & 13920 & Drift-Gas \\
{$[7]$} & 2 & 784 & 1852 & Face Recognition \\
\hline
\end{tabular}

In this experiment excluding for data face, classes multiple and features greater of 600 datasets rest. Methodology proposed begin in layer one where neurons selected randomly in this layer, function fitness calculation following by the best solution which represent initial solution then starts solution optimal global searching from here. $B \max =20$ for every iteration and Iter=10 by iterated each solution selected. Additional into two parts divided of Bmax, one part in layer selected neurons number increases and the same decreasing at second part. The $3 \%$ set of $\omega$ neurons up edition and the value of 0.5 set layer particular neurons changing probability and maximum layers runs by this methodology is Kmax equal to 5 layers and if needed more can increased. SOFM algorithm proposed executed for error testing and training terms of different $\mathrm{KL}=\{1,2,3,4,5\}$ shown in Figure 6 (a) and (b).

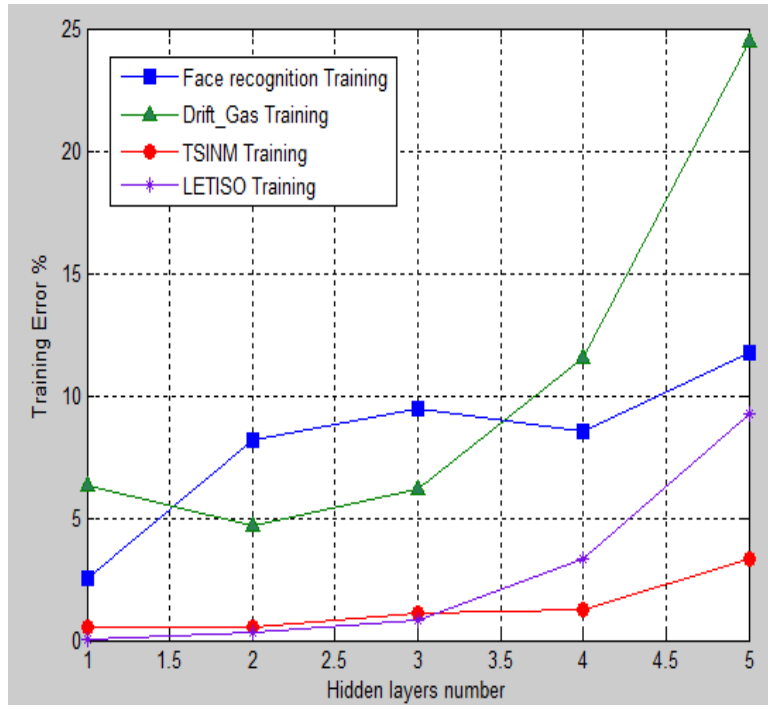

(a)

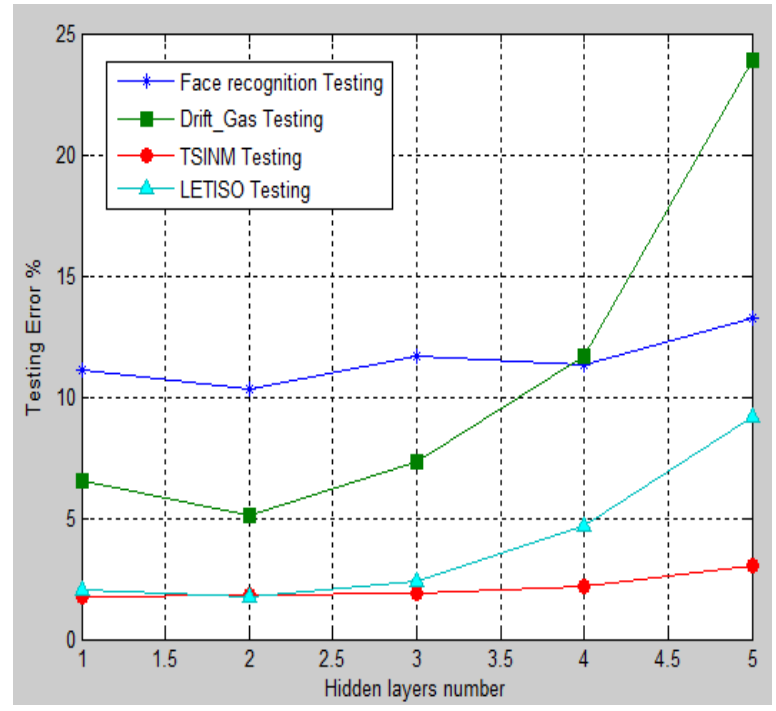

(b)

Figure 6. Algorithm proposed performance for various hidden layers applied in,

(a) Training error, (b) Testing error 
Table 2 shown methodology proposedresults, approach proposed according architecture optimized datasets face recognition was $\mathrm{KL}=2$ and $\mathrm{KM}=(437,260)$ with $10.34 \%$ error testing classification. The optimal topology dataset of drift gas was $\mathrm{KL}=2$ and $\mathrm{KM}=(91,63)$ with $5.073 \%$ error testing classification. The best topology dataset in TSINM registed $\mathrm{KL}=1$ and $\mathrm{KM}=517$ with $1.745 \%$ error testing classification While architecture optimal topology for dataset LETISO requires $\mathrm{KL}=2$ and $\mathrm{KM}=(363,232)$ with $1.765 \%$ error testing classification.

Table 2. Results of experimental collected by proposed method

\begin{tabular}{lcclc}
\hline Datasets & Testing Error & Training Error & Neurons Hidden & Hidden Layer \\
\hline LETISO & 2.0698 & 0.0301 & 335 & 1 \\
& $\mathbf{1 . 7 6 5 8}$ & 0.3378 & $\mathbf{3 6 3 , 2 3 2}$ & $\mathbf{2}$ \\
& 2.3935 & 0.8519 & $397,212,119$ & 3 \\
& 4.6555 & 3.3127 & $406,254,155,100$ & 4 \\
TSINM & 91.484 & 92.448 & $402,227,135,78,46$ & 5 \\
& $\mathbf{1 . 7 4 5}$ & 0.521 & $\mathbf{5 1 7}$ & $\mathbf{1}$ \\
& 1.832 & 0.545 & 548,224 & 2 \\
& 1.917 & 1.1127 & $521,289,165$ & 3 \\
Drift_Gas & 2.155 & 1.2714 & $532,302,130,75$ & 4 \\
& 3.053 & 3.3475 & $490,274,158,103,64$ & 5 \\
& 6.5471 & 6.3171 & 72 & 1 \\
Face & $\mathbf{5 . 0 7 3 9 8}$ & 4.6761 & $\mathbf{9 1 , 6 3}$ & $\mathbf{2}$ \\
recognition & 7.2962 & 6.1466 & $\mathbf{7 9 , 5 2 , 3 7}$ & 3 \\
& 11.7081 & 11.5086 & $75,78,34,23$ & 4 \\
& 23.8846 & 24.4556 & $76,42,36,22,18$ & 5 \\
& 11.133 & 2.5125 & 515 & 1 \\
& $\mathbf{1 0 . 3 4 2 1}$ & 8.1481 & $\mathbf{4 3 7 , 2 6 0}$ & $\mathbf{2}$ \\
& 11.6585 & 9.4384 & $506,261,140$ & 3 \\
& 11.3072 & 8.5615 & $454,261,148,73$ & 4 \\
\hline
\end{tabular}

\section{CONCLUSIONS}

Architecture deep FNN optimizing applied successfully in SOFM with proposed algorithm as shows in this work and got optimal solution, space searching entity considered testing error, hidden neurons and hidden layers represented solution. The good performance with testing error lowest of FNN solution optimal global found is methodology aim. Samples and attributes of huge number in experiment used as dataset. Methodology proposed by suggested deep FNN architecture show generated result. High accuracy getting for every dataset of architecture FNN required two hidden layers methodology proposed, excluding dataset of TSINM finding interesting. Therefore greater of 1 hidden layer requires FNN where cases in work can methodology proposed such that networks neural deep applied. Future work solution same in connections optimal find extended further can be work and methodology proposed based on approach hybrid develop combined such as PSO,GA and SA techniques that have optimization solutions.

\section{REFERENCES}

[1] Forest Agostinelli, Michael R. Anderson, Honglak Lee, "Adaptive multi-column deep neural networks with application to robust image denoising," in Advances in Neural Information Processing Systems, pp. 1493-1501, 2013.

[2] W. Yang, Y. Tian, F. Zhou, Q. Liao, H. Chen and C. Zheng, "Consistent Coding Scheme for Single-Image Super-Resolution Via Independent Dictionaries," in IEEE Transactions on Multimedia, vol. 18, no. 3, pp. 313-325, 2016.

[3] C. Ledig et al., "Photo-Realistic Single Image Super-Resolution Using a Generative Adversarial Network," 2017 IEEE Conference on Computer Vision and Pattern Recognition (CVPR), Honolulu, HI, pp. 105-114, 2017.

[4] D. Marín, A. Aquino, M. E. Gegundez-Arias and J. M. Bravo, "A New Supervised Method for Blood Vessel Segmentation in Retinal Images by Using Gray-Level and Moment Invariants-Based Features," in IEEE Transactions on Medical Imaging, vol. 30, no. 1, pp. 146-158, Jan. 2011.

[5] A,Vergara, et al., "Chemical gas sensor drift compensation using classifier ensembles," Sensors Actuators $B$ Chemical, vol. 166-167, pp. 320-329, 20 May 2012.

[6] D. Cascio et al., "Mammogram Segmentation by Contour Searching and Mass Lesions Classification With Neural Network," in IEEE Transactions on Nuclear Science, vol. 53, no. 5, pp. 2827-2833, Oct. 2006.

[7] M. Da'san, A. Alqudah and O. Debeir, "Face detection using Viola and Jones method and neural networks," 2015 International Conference on Information and Communication Technology Research (ICTRC), Abu Dhabi, pp. 40-43, 2015.

[8] P. Kamencay, D. Jelšovka and M. Zachariasova, "The impact of segmentation on face recognition using the principal component analysis (PCA)," Signal Processing Algorithms, Architectures, Arrangements, and Applications SPA 2011, Poznan, pp. 1-4, 2011. 
[9] Daniel Madan Raja S,A Shanmugam, "ANN and SVM Based War Scene Classification using Wavelet Features:A Comparative Study," Journal of Computational Information Systems, vol. 7 no. 5, 2011.

[10] M. Riedmiller and H. Braun, "A direct adaptive method for faster backpropagation learning: the RPROP algorithm," IEEE International Conference on Neural Networks, San Francisco, CA, USA, vol.1, pp. 586-591, 1993

[11] Y. Xia and M. S. Kamel, "Novel Cooperative Neural Fusion Algorithms for Image Restoration and Image Fusion," in IEEE Transactions on Image Processing, vol. 16, no. 2, pp. 367-381, Feb. 2007.

[12] Jiaying Deng, Wenhai Zhang, Xiaomei Yang, "Recognition and Classification of Incipient Cable Failures Based on Variational Mode Decomposition and a Convolutional Neural Network," Energies, vol. 12, no. 10, pp. 1-16, 2019.

[13] O. Janssens, et al.,"Convolutional neural network-based fault detection for rotating machinery," Journal of Sound and Vibration, vol. 377, pp. 331-345, September 2016.

[14] B. Al-kazemi and C. K. Mohan, "Training feedforward neural networks using multi-phase particle swarm optimization," Proceedings of the 9th International Conference on Neural Information Processing, 2002. ICONIP '02., Singapore, vol. 5, pp. 2615-2619, 2002.

[15] G. E. Dahl, D. Yu, L. Deng and A. Acero, "Context-Dependent Pre-Trained Deep Neural Networks for Large-Vocabulary Speech Recognition," in IEEE Transactions on Audio, Speech, and Language Processing, vol. 20, no. 1, pp. 30-42, Jan. 2012.

[16] S. Aydin and H. Temeltas, "Time optimal trajectory planning for mobile robots by differential evolution algorithm and neural networks," 2003 IEEE International Symposium on Industrial Electronics (Cat. No.03TH8692), Rio de Janeiro, Brazil, vol. 1, pp. 352-357, 2003.

[17] F. Seide, G. Li, X. Chen and D. Yu, "Feature engineering in Context-Dependent Deep Neural Networks for conversational speech transcription," 2011 IEEE Workshop on Automatic Speech Recognition \& Understanding, Waikoloa, HI, pp. 24-29, 2011.

[18] S. Li and A. Chan,"3D human pose estimation from monocular images with deep convolutional neural network," In Asian Conference on Computer Vision, Springer: Cham, Switzerland,2017, pp. 332-347.

[19] H. Jung, M. Choi, K. Soon and W. Y. Jung, "End-to-end pedestrian collision warning system based on a convolutional neural network with semantic segmentation," 2018 IEEE International Conference on Consumer Electronics (ICCE), Las Vegas, NV, pp. 1-3, 2018.

[20] Hiroki Yoshimura, Tadaaki Shimizu, Toshie Matumura(Nara National College), Masaya Kimoto, Naoki Isu, "Adaptive Noise Reduction Filter for Speech Using Cascaded Sandglass-Type Neural Network", ITC-CSCC:International Technical Conference on Circuits Systems, Computers and Communications, pp. 605-608, 2008.

[21] Mohamad Aqil Mohd Fuad, Mohd Ruddin Ab Ghani, Rozaimi Ghazali, Tarmizi Izzuddin, "Training of Convolutional Neural Network using Transfer Learning for Aedes Aegypti Larvae," TELKOMNIKA Telecommunication Computing Electronics and Control,vol.16,no.4,pp. 1894-1900, August 2018.

[22] Parinith R Iyer, Shrutheesh Raman Iyer, Raghavendran Ramesh, Anala M. R., K. N. Subramanya, "Adaptive real time traffic prediction using deep neural networks," International Journal of Artificial Intelligence (IJ-AI), vol. 8, no. 2, pp. 107-119, June 2019.

[23] Adel Ahmed Obed, Ameer Lateef Saleh, Abbas Kareem Kadhim, "Speed performance evaluation of BLDC motor based on dynamic wavelet neural network and PSO algorithm," International Journal of Power Electronics and Drive System, vol. 10, no. 4, pp. 1742-1750, December 2019.

[24] Hilman F. Pardede, Asri R. Yuliani, Rika Sustika, "Convolutional Neural Network and Feature Transformation for Distant Speech Recognition," International Journal of Electrical and Computer Engineering, vol. 8, no. 6, pp. 5381-5388, December 2018.

[25] Muthna Jasim Fadhil, Maitham Ali Naji, Ghalib Ahmed Salman, "Transceiver error reduction by design prototype system based on neural network analysis method," Indonesian Journal of Electrical Engineering and Computer Science, vol. 18, no. 3, pp. 1244 1251, June 2020.

[26] Chan Hong Goay, Azniza Abd Aziz, Nur Syazreen Ahmad, Patrick Goh, "Progress in neural network based techniques for signal integrity analysis-a survey," Bulletin of Electrical Engineering and Informatics, vol. 8, no. 1, pp. 276-282, March 2019.

[27] Ngoc Thuy Pham, Diep Phu Nguyen, KhuongHuu Nguyen, Nho Van Nguyen, "New Version of Adaptive Speed Observer based on Neural Network for SPIM," International Journal of Power Electronics and Drive System, vol. 9, no. 4, pp. 1486-1502, December 2018.

[28] Soukaina Barhmi, Omkalthoume El Fatni, "Hourly wind speed forecasting based on support vector machine and artificial neural networks," International Journal of Artificial Intelligence, vol. 8, no. 3, pp. 286-291, September 2019. 